\title{
Intestinal T-Cell Lymphoma
}

National Cancer Institute

\section{Source}

National Cancer Institute. Intestinal T-Cell Lymphoma. NCI Thesaurus. Code C150495.

A group of mature T-cell and NK-cell non-Hodgkin lymphomas that includes

enteropathy-associated T-cell lymphoma, monomorphic epitheliotropic intestinal T-cell

lymphoma, and intestinal T-cell lymphoma, not otherwise specified. Most of these

lymphomas arise from the small intestine and a minority from the large intestine or stomach. 